\title{
Research on Properties of Functional Fabrics in Home Textiles based on Blue Calico
}

\author{
Lei Zhang ${ }^{1, a}$, Kehui Deng ${ }^{2, b}$ and Ziqi Wang ${ }^{2, c}$ \\ ${ }^{1}$ College of Textiles, Donghua University, Shanghai201620, China;College of Electronic and \\ Electrical Engineering, Wuhan Textile University, Wuhan430200, China \\ ${ }^{2}$ College of Humanities, Donghua University,201620, China \\ a282526405@qq.com, bdengkh@dhu.edu.cn,,390166030@qq.com
}

Keywords:Blue Calico;Doubling Yarn;Plied Yarn;Breaking Strength;Abrasion Resistance; Pilling Resistance; Air Permeability; Friction

Abstract: In this paper, we take an innovative experiment on one fabric in home textiles based on Blue Calico, to study the plying structure of yarns and properties of the fabric. Under the circumstance of the same warp yarn, we compare these different fabric weaved by a plurality of high count plying yarns without twisting, the same plying yarns with twisting, and the same single yarns as the weft yarns, then to research advantages and disadvantages of the plying yarns with twisting or without twisting and its impact on home textiles.

\section{Introduction}

The development of textile industry in the 21 st century brings about demands of newer and more humanized designs in home textiles[1]. At present, the most famous of China's existing blue calico of starching dye-resist is Nantong's blue calico, and in June 2006 it was selected as the first batch of national intangible cultural heritage [2].As people's living standards continue to improve and demands continue to deepen, the textile fabrics of high count plying yarns with twisting are more and more in the market, while fabrics of plying yarns without twisting are in its infancy, so the research for this application has important theoretical values and practical significances.

\section{Materials and methods}

In the experiment, we choose polyesters as the warp yarn, cotton plied yarns and cotton doubling yarns as the weft yarn, besides the cotton doubling yarns is a plurality of yarns together without twisting; we choose five with satin weave of three fly, while the selvedge with plain weave of two on and two under. Fabrics' specifications are as follows:

Yarns material: Polyesters Reed number: 2 Counts of the warp yarn: $25 \mathrm{Ne}$

Number of ground warp yarns: $480 \quad$ Number of side warp yarns: 24

Width of the fabric: $20 \sim 21(\mathrm{~cm})$

This article is the research and study through comparing data and testing properties of fabrics of high count doubling yarns and fabrics of the same plied yarns. First, determining the desired counts of yarns; second, spooling yarns with doubling and plying respectively; then, weaving it on a small model loom; finally, researching doubling fabrics through a series of tests of anti-bending, brute force, friction, pilling, draping. 


\section{Results and discussion}

\section{Appearances and parameters of fabrics}

The density test of Fabrics' warp yarns and weft yarns: under the same condition of weaving, taking two pieces of cloth woven separately, and direct reading with the mobile instrument of fabrics' density's measuring. The distance to measure is $5 \mathrm{~cm}$, each group with 4 times' data and calculating the average.

Table 1 Data sheet of density Unit: root/ $5 \mathrm{~cm}$

\begin{tabular}{ccccccc}
\hline Fabrics' types & Direction & 1 & 2 & 3 & 4 & Average \\
\hline Fabrics of doubling yarns & $\mathrm{P} \mathrm{j} / 5 \mathrm{~cm}$ & 120 & 115 & 117 & 115 & 116.75 \\
& $\mathrm{P}_{\mathrm{w} / 5 \mathrm{~cm}}$ & 128 & 132 & 127 & 125 & 128 \\
Fabrics of plying yarns & $\mathrm{P} \mathrm{j} / 5 \mathrm{~cm}$ & 118 & 119 & 117 & 119 & 118.25 \\
& $\mathrm{P}$ w/5 cm & 128 & 134 & 132 & 136 & 132.50 \\
\hline
\end{tabular}

From the data in Table 1 ,we can see that the yellow sample's density (fabrics of plying yarns) is slightly larger than the red sample's (fabrics of doubling yarns). But from the hand feeling of these two samples, the yellow one is relatively softer.

\section{The comparative test of fabrics' tensile breaking}

The tensile breaking strength of the fabric is used to indicate the ability to resist tensile forces, and also used to assess changes before and after wear and tear, in order to evaluate the effects of sunshine, washing and various finishing on the intrinsic quality of the fabric[3].Testing with the YG(B) 026-250 electronic tester of fabric strength, using the method of splitting selvage of fabrics, and setting the clamping distance as $100 \mathrm{~mm}$. Stretching the samples of a certain size to break them according to a constant elongation. Each sample cloth is tested for two times (the weft direction).

Table 2 Data sheet of fabrics' tensile failure

\begin{tabular}{lllll}
\hline Types Direction & \multicolumn{5}{c}{ Breaking Force Breaking Elongation Braking Time Elongation } \\
& $\mathrm{F}(\mathrm{N})$ & $\mathrm{L}(\mathrm{mm})$ & $\mathrm{T}(\mathrm{S})$ & $\%$ \\
\hline Fabrics of doubling yarns & Weft 1251.8 & 14.005 & 7.975 & 14.01 \\
Fabrics of plying yarns & Weft 622.75 & 16.9 & 6.795 & 16.90 \\
\hline
\end{tabular}

As can be seen from Table 2, the breaking force in the weft direction of fabrics of plying yarns is smaller by $50.25 \%$ than that of fabrics of doubling yarns, breaking elongation of which is higher by $20.67 \%$ than that of fabrics of doubling yarns. Although the density of fabrics of plying yarns is slightly larger than that of doubling yarns, the breaking strength of which is smaller; this may have a close relationship with its single yarn's strength. The experimental result is: the breaking force in the weft direction of fabrics of doubling yarns is better than that of fabrics of plying yarns. It shows that the fabric of doubling yarns is more stretch-proof in the direction of its weaving.

\section{The test of fabrics' abrasion resistance}

Fabrics would cause friction with people's skins or clothes during wearing. Studies have shown that $7 \%$ of clothes' damage is due to wear and tear. The slippage of fabrics' yarns, especially at the place of interweaving, is connected to the property of fabrics' abrasion resistance [4]. Testing with the Y522 type disc grinding apparatus, and the sample is divided into two groups, one group is fabrics of doubling yarns, and another is fabrics of plying yarns. Abrasive is the A-150 charring wheel, the pre-tension of the pressurized hammer is $250 \mathrm{~g}$, while the number of turns is 200 times. 
Table 3 Testing data of abrasion

\begin{tabular}{ccccc}
\hline Types & $\begin{array}{c}\text { Weighing } \\
\text { Time }\end{array}$ & $\begin{array}{c}\text { Sample } \\
\text { Weight }(\mathrm{g})\end{array}$ & $\begin{array}{c}\text { Wearing } \\
\text { Weight }(\mathrm{g})\end{array}$ & $\begin{array}{c}\text { Weight Loss/ } \\
\text { Unit Areag/(cm 2) }\end{array}$ \\
\hline $\begin{array}{c}\text { Fabrics of } \\
\text { doubling yarns }\end{array}$ & $\begin{array}{c}\text { Pre-wearing } \\
\text { Post-wearing }\end{array}$ & 3.2968 & 0.0267 & 0.000218 \\
$\begin{array}{c}\text { Fabrics of } \\
\text { plying yarns }\end{array}$ & Pre-wearing & 2.6448 & 0.0354 & 0.000289 \\
\hline
\end{tabular}

As can be seen from the Table 3, under the same wearing condition, the weight loss per unit area of fabrics of plying is higher by $32.57 \%$ than that of fabrics of doubling yarns. This experiment shows that the abrasion resistance of fabrics of doubling yarns is better than that of fabrics of plying yarns.

\section{The comparison test of fabrics' pilling resistance}

Fabrics constantly expose to various external forces in the process of using that makes fluff or monofilament on the surface of fabrics gradually be pulled out. When the height and density of fluff reaches a certain value, the continuous effect of external friction makes the fluff tangled into a ball and emboss on the surface of the fabric. The pilling of the fabric will deteriorate the appearance of the fabric, reducing its wearability.

Testing with YG502 fabrics' pilling tester, and dividing the sample into two groups, one group is the fabric of doubling yarns, and another is the fabric of plying yarns. Cutting a sample with the diameter of $(113 \pm 0.5)$ for each group, with nylon brushing abrasive and the standard fabric's abrasive, using the testing method of circular locus. The pre-pressure is $300 \mathrm{CN}$; nylon abrasive grinds for 150 times, and the fabric's abrasive grinds for 150 times.

Table 4 The comparison sheet of pilling of fabrics' types

\begin{tabular}{ccc}
\hline Fabrics' types & Fabrics'directions & Levels of samples \\
\hline Fabrics of doubling yarns & Opposite & Second \\
Fabrics of plying yarns & Negative & First \\
\hline
\end{tabular}

As can be seen from Table4, fabrics of doubling yarns is the second level comparing to the standard level of samples, and fabrics of plying yarns is the first level comparing to the standard level of samples. It shows that the pilling resistance of fabrics of doubling yarns is better than that of fabrics of plying yarns.

\section{The comparative test of fabrics' air permeability}

Fabrics' ability to penetrate the air is important to garments. Outerwear in winter should be windproof and warm, namely it should have less air permeability; garments in summer should have a better air permeability for a cool feeling.Testing with YG (B) 461D type - II digital instrument of fabrics' air permeability, with the 3rd nozzle, and the range of dynamic pressure is $600 \sim 3000 \mathrm{~Pa}$, the sample's pressure is $100 \mathrm{~Pa}$, the sample area is $20 \mathrm{~cm}^{2}$.

Table 5 Data sheet of fabrics' air permeability Unit: $\mathrm{L} / \mathrm{m}^{2} \mathrm{~s}$

\begin{tabular}{lccccccc}
\hline \multicolumn{1}{c}{ Samples } & 1 & 2 & 3 & 4 & 5 & 6 & Average \\
\hline Fabrics of doubling yarns & 211.2 & 214.7 & 147.6 & 172.1 & 162.1 & 222.3 & 188.3 \\
Fabrics of Plying yarns & 216.8 & 111.3 & 233.0 & 213.4 & 140.3 & 149.4 & 177.4 \\
\hline
\end{tabular}

Under the circumstance of the same intensity of pressure, the property of fabrics' air permeability is mainly related to the internal structure of fabrics. The pore size of the fabric is apparently 
negatively related to the tightness of the fabric and the length of the tissue's float, but it also has some relevance with the thickness of the fabric [5]. As shown in Table5, the air permeability of fabrics of doubling yarns is higher by $6.1 \%$ than that of fabrics of plying yarns, which is the air permeability of fabrics of doubling yarns is apparently better than that of fabrics of plying yarns.

\section{Conclusions}

This paper focuses on the property of the high count doubling fabric in Home Textiles based on Blue Calico, through referring to the experiments, we find that the physical property of high count doubling fabrics is better than that of fabrics of plied yarns, such as properties of air permeability, pilling resistance, abrasion resistance and strength against breaking and so on; although the outlook and hand feeling of which is not as good as that of fabrics of plied-yarns, while the practical value of the former is of higher. If this kind of fabric is used in bedding and home textiles, it will be more satisfied with the need of consumers no matter in respect of its appearance or its properties; what's more, this kind of fabric will be easier to have a wider prospect in the field of home textiles.

\section{References}

[1]N. Liu: Foreign Silk.Vol.2(2005),p.25 (In Chinese)

[2]Y.X.Wu,in:Blueprint Cloth, edtied by Jiangsu Arts Press,Jiangsu,NY(2014), in press.

[3]J.Li,T.Y.Zheng,J.X.Yang,et al:TianjinTextile Institute of Technology, Vol.4(1998),p. 92 (In Chinese)

[4]B.X.Cai.The Structures and Designs of Fabrics, edtied by China Textile Press, NY(2008), in press.

[5]M.W. Qiu, F.M. Wang:Textile Technology, Vol.8(2005),p.73 (In Chinese) 